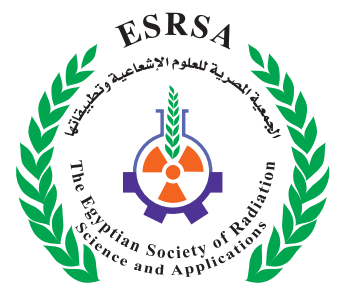

J. Nucl. Tech. Appl. Sci., Vol. 8, PP. 145 : 152 (2020)

\title{
Assessment of Level of Collimation for Pediatric Plain Chest \\ Radiographs in a Teaching Hospital in Kano, Northwestern Nigeria
}

Joseph, D.Z. ${ }^{1}$; Amina, A.M ${ }^{1}$; Umar, M.S ${ }^{1}$; Sidi, $M^{1}{ }^{1}$; Shem, B. S. ${ }^{1}$, Nkubli, F.B. ${ }^{2}$

Received: 13/02/2020

Accepted: 18/10/2020

DOI: $10.21608 /$ jntas.2020.23934.1017

E.mail:umar.sani888@gmail.com

\section{KEYWORDS}

Collimation, Pediatric, Radiation Protection, Image Quality, Chest Radiography.

\section{ABSTRACT}

Collimation of x-ray beam is an important factor in optimization; good collimation will both minimize the radiation dose to the patient and improve image quality because the amount of scattered radiation will increase as if a large volume of tissue is irradiated. This study aimed to assess the level of collimation for pediatric plain chest radiographs in Aminu Kano Teaching Hospital as an optimization tool. A retrospective audit was conducted on pediatric plain chest radiographs from May 2016 to May 2019 in Aminu Kano Teaching Hospital (Kano, Nigeria) using a simple random sampling technique. Data were collected and recorded using self-developed data capture sheet based on the European Guidelines on quality criteria for diagnostic radiographic images in pediatrics which captures the gender, age, projection, collimation (4 ways, 3 ways, 2 ways, 1 way, no and under collimation). The results showed that the most predominant age group exposed in the study was within 0 to 1 year representing 31.7\% $(n=76)$. Also gender distribution showed that more male were exposed than female, representing $55 \%(n=132)$. Out of 240 plain chest radiographs, about $77.0 \%(\mathrm{n}=175)$, showed inadequate collimation. In conclusion, the study revealed poor collimation practice in pediatric plain chest radiographs.

1. Department of Radiography, Bayero University Kano-Kano, Nigeria.

2. Department of Medical Radiography, University of Maiduguri, Borno-Nigeria. 


\section{INTRODUCTION}

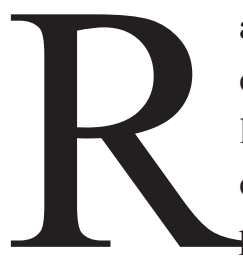

adiographic imaging of the pediatric chest presents several unique challenges and nuances, stemming from congenital variants and pathologic Errors in interpretation may further lead to inappropriate imaging, incurring additional radiation exposure and cost, as well as to psychological effects on the patients and their families (Menashe et al., 2016). Chest radiography is one of the most widely used diagnostic examinations for children (Stollfuss et al., 2015). In a special care baby unit, premature neonates have serious and life-threatening diseases that may require a large number of x-rays for diagnosis and treatment. Increased neonatal radiosensitivity and longer life expectancy increase the risk of radiation-induced cancer, which emphasizes the importance of minimizing the dose while maintaining clinically satisfactory image quality (Stollfuss $\boldsymbol{e t}$ al., 2015).

Conventional chest radiography is a proven and useful procedure for imaging of the main airways, lungs, mediastinum, heart, pleura and chest wall (Duwlfoh, 2013). The basic view is the postero-anterior (PA) of the chest in an upright position. The role and importance of the radiograph's quality are to help the radiologists and the clinicians in the diagnosis of diseases and its management. Thus it is important to show the entire anatomical structure, respiration accuracy by counting six anterior ribs/ ten posterior ribs, adequate penetration to see the lower thoracic intervertebral discs through radiographs by evaluating the quality of depiction of the anatomical and physical details (Duwlfoh, 2003).

The most essential difference between adult and pediatric radiography is the different disease spectrum of children and the resulting clinical questions regarding imaging. Together with efforts to perform diagnostic procedures that are as gentle but as reliable as possible, strict indication guidelines for chil- dren are one of the most essential factors in radiation protection (Alzen \& Benz-Bohm, 2011). The radiation concerns are significantly more for the pediatric population, as this group is particularly vulnerable to harmful effects of the radiation, mainly due to rapidly dividing cells in their growing bodies which are more radio-sensitive, and also due to longer life expectancy of the children, which may allow potential oncogenic radiation effects to manifest themselves at a later date (Gupta \& Upreti, 2017).

Collimation of the x-ray beam is an important factor in optimization. Good collimation will both minimize the dose to the patient and improve image quality because the amount of scattered radiation will increase as if a large volume of tissue is irradiated. Collimation is particularly important in pediatric radiography since the organs are closer together and larger fields are more likely to include additional radiosensitive organs (Nkubli et al., 2016).

Collimation is one of the most effective radiation protection measures for the patient and personnel because it reduces the area of radiation coverage. It brings about an effective dose reduction and also produces better image quality. Collimation is performed using cones and collimators. Other methods of limiting x-ray exposure to the patient include the use of shields, for example a gonad shield (Moi $\boldsymbol{e t}$ al., 2017). Collimation decreases a patient's radiation dose by limiting x-ray exposure to the area of interest. It also has the added benefit of improving the quality of the resulting image. It is the restriction of x-radiation to the area being examined or treated by confining the beam with a metal diaphragm with high radiation absorption power (Moi et al., 2017).

Collimation in most cases depends on the technique of the radiographer, but regular quality assurance by checking that the X-ray beam and the field from the light beam diaphragm are accurately aligned is important, particularly for mobile equipment (Martin, 2007). However, this study aims to evaluate the adequacy of collimation as a radiation 
protection measure among plain pediatric chest radiographs in Aminu Kano Teaching Hospital, from Kano, Nigeria.

\section{MATERIALS AND METHODS.}

This is a retrospective cross-sectional study carried out among pediatric patients referred for a plain chest x-ray in Aminu Kano Teaching Hospital from January 2016 to May 2019. Aminu kano teaching hospital is a fully five hundred-bed tertiary government teaching hospital located in kano, kano state, Nigeria. the hospital was established in august, 1988 formerly known as Aminu Kano cottage hospital. the hospital has about twenty-three departments. the radiology department is one the major departments in the hospital which has about 100 to 150 patients through per day. the department comprises of so many units which includes but not limited to: two conventional radiography units, two CT scans, three fluoroscopy machines, one mammography unit, one angiography (interventional) suites, one Direct Radiography unit among others. The data were collected and recorded using self-developed data capture sheet based on European Guidelines on Quality Criteria for Diagnostic Radiographic Images in Pediatrics which contains a column for gender, age, projection, collimation (4 ways, 3 ways, 2 ways, 1 way, no and under collimation).plain chest radiographs were acquired using standard dedicated pediatric radiography unit with exposure of factors ranging between 60-54 peak kilovoltage on 5-8miliampere seconds. Most images were acquired with patients in supine position (Anterior-posterior projection) with horizontal x-ray beam and very few in erect standing position (posterior-anterior projection) with vertical x-ray beam under deep arrested inspiration technique. Immobilization tools were used at some instances. All pediatric patients undergoing plain chest radiographs at Aminu Kano Teaching Hospital in Kano were selected at random from January 2016 to May 2019. However, all non-pediatric plain chest radiographs in the study center and all pediat- ric and non-pediatric radiographs outside the center of study were excluded from the study. Statistical package for social sciences (SPSS Chicago version 23.0) was used for analysis, where mean frequencies and percentages were obtained. In line with Helsinki declaration, institutional review board approval was obtained from Health Research Ethics Committee of Aminu Kano Teaching Hospital, and all information obtained were treated with utmost confidentiality.

\section{RESULTS AND DISCUSSION}

Table 1 summarizes the age and gender distribution of patients who underwent plain chest radiographs in the study. Out of the 240 pediatric plain chest radiographs ( $\leq 16$ years) assessed for collimation as a means of radiation protection practice based on European guidelines. The children from the age group 0-1 years (infants) were the most involved in the study, representing about $31.7 \%$ (76 patients) . The proportion of sex distribution in the pediatric plain chest radiographs was 132 males $(55.0 \%)$ and 108 females $(45.0 \%)$.

Tables 2 and 3 summarize the collimation adequacy and level of collimation of pediatric plain chest radiographs based on the presence or absence of silver linings. It is clearly seen that majority of the radiographs (175 radiographs, representing 73.0\%) were inadequately collimated. The total of adequately collimated radiographs with silver linings presence on four (4), three (3) and two (2) sides was: $16(6.7 \%), 15(6.3 \%)$ and $34(14.2 \%)$, respectively from the total. The total number of non-collimated radiographs, under collimated radiographs and silver linings presence on one (1) way side was: 118 $(49.2 \%), 4(1.70 \%)$ and $53(22.1 \%)$, respectively from the total.

Proper collimation to the area of interest under investigation is one of the ways to avoid unnecessary exposure of patients to ionizing radiation, hence the need to assess the level of collimation to avoid harming the pediatric population. Moreover, inad- 
equate collimation among pediatric patients could affect image quality and can lead to high radiation dose to pediatrics which could lead to deleterious effects. The collimator sometimes referred to as the light beam diaphragm (LBD) provides the radiographer with an easy method of limiting the size of the $\mathrm{x}$-ray field and placing it over the area of interest, thus reducing the radiation dose to the patient and improving the quality of the image. The collimator receives much use and is vulnerable to knocks often resulting in the inaccuracy of the light beam coincidence blown light bulbs, electrical and mechanical problems (Peter, 2004).

Table (1): The age and gender distribution of the pediatric plain chest radiographs.

\begin{tabular}{|c|c|c|}
\hline AGE GROUP (YEARS) & FREQUENCY (N) & PERCENTAGE (\%) \\
\hline $0-1$ & 76 & 31.7 \\
\hline $2-6$ & 69 & 28.8 \\
\hline $7-11$ & 42 & 17.5 \\
\hline $12-16$ & 53 & 22.1 \\
\hline TOTAL & $\mathbf{2 4 0}$ & $\mathbf{1 0 0 . 0}$ \\
\hline GENDER & & 55.0 \\
\hline MALE & 132 & 45.0 \\
\hline FEMALE & 108 & $\mathbf{1 0 0 . 0}$ \\
\hline TOTAL & $\mathbf{2 4 0}$ & \\
\hline
\end{tabular}

Table (2) : Collimation adequacy based on the presence of silver lines.

\begin{tabular}{|c|c|c|}
\hline OVERALL BEAM COLLIMATION & FREQUENCY & PERCENTAGE \\
\hline ADEQUATE & 65 & $27.0 \%$ \\
\hline INADEQUATE & 175 & $73.0 \%$ \\
\hline TOTAL & 240 & $100.0 \%$ \\
\hline
\end{tabular}

Table (3) : The level of collimation of the pediatric plain chest radiographs.

\begin{tabular}{|c|c|c|}
\hline LEVEL OF COLLIMATION & FREQUENCY & PERCENTAGE \\
\hline $\mathbf{4}$ WAY & 16 & $6.7 \%$ \\
\hline $\mathbf{3}$ WAY & 15 & $6.3 \%$ \\
\hline $\mathbf{2}$ WAY & 34 & $14.2 \%$ \\
\hline $\mathbf{1}$ WAY & 53 & $22.1 \%$ \\
\hline NON & 118 & $49.2 \%$ \\
\hline UNDER & 4 & $1.70 \%$ \\
\hline TOTAL & $\mathbf{2 4 0}$ & $\mathbf{1 0 0 . \%}$ \\
\hline
\end{tabular}


240 paediatric plain chest radiographs were evaluated based on the presence of silver linings, as follows: $6.7 \%$ (16 patients) had silver linings on four sides, $6.3 \%$ (15 patients) had silver linings on three sides, $14.2 \%$ (34 patients) had silver linings on two sides, $22.1 \%$ (53 patients) had silver linings on one side, $29.2 \%$ (118 patients) has no evidence of silver linings and $1.7 \%$ (4 patients) are under collimated. The study shows that $23.0 \%$ (65 patients) of the total chest radiographs were adequately collimated, the rest of $77.0 \%$ (175 patients) being inadequately collimated. This study is in line with findings of a study (Okeji et al., 2017) which revealed $82.0 \%$ of poor collimation practice. However, the study also considered other studies (Nkubli et al., 2016) on radiation protection practice on pediatric chest radiographs using beam collimation as a criterion which showed that $79.0 \%(n=158)$ of the radiographs have evidence of adequate beam collimation. This may be due to the fact that most of radiographers do not regard under collimation as an issue of concern when judging image quality based on collimation. Another similar study (Moi et al., 2017) was carried out and found that radiation protection to the patient concerning collimation on plain chest radiographs is adequate. This study also disagrees with the current study.

It was also found out that the patients within 0 -1years, representing about $31.7 \%$ from the total, were the most exposed category, being mostly referred from the special care baby unit (SCBU); this may be attributed to investigation to exclude congenital anomaly at the time of birth found within the age group.

\section{CONCLUSION}

This study revealed poor collimation practice in chest radiography. With more males' patients exposed than females. The most exposed age group in pediatric for plain chest radiographs was the group with $\leq 1$ year of age. Hence, the need to carry out regular light beam alignment tests on x-ray ma- chines, regular training and retraining of radiographers on pediatric radiation protection measures. No conflict of interest.

\section{REFERENCES}

- Alzen, G. \& Benz-Bohm, G. (2011): Radiation Protection in Pediatric Radiology. Dtsch. Arztebl. Int., 108(24): 407.

- Duwlfoh, U. (2003): Evaluation of image quality in chest radiographs. J. Inst. Med., 3.

- Gupta, N. \& Upreti, L. (2017): Optimal Utilization of Pediatric Computed Tomography to Minimize Radiation Exposure: What the Clinician Must Know. Indian Ped., 54: 581.

- Martin, C. (2007): Optimization in general radiography. Biomed. Imaging Interv. J., 3(2): e18.

- Menashe, S.J.; Iyer, R.S.; Parisi, M.T.; Otto, R.K. and Stanescu, A.L. (2016): Pediatric Chest Radiographs: Common and Less Common Errors. Am. J. Roentgenol., 207(4): 903.

- Moi, A.S.; Ado, Z.; Zira, J.D.; Shem, S.; Ginkanwa, N.; Malgwi, F. and Umar, M.S. (2017): Assessment of Collimation on Adult Plain Chest Radiographs as a Radiation Protection Measure in a Nigerian Teaching Hospital. Pac. J. Sci. Technol., 18(1): 308.

- Nkubli, F.B.; Moi, A.S.; Bashir, A.G.; Ivor, C. and Nzotta, C.C. (2016): Original Article Radiation Protection Practice on Paediatric Chest Radiographs Using Beam Collimation As A Criterion. Pak. J. Radiol., 26(4): 317.

- Okeji, M.; Agwuna, K.; Abubakar, U.; Izge, I.; Aninworie, A. and Arogundade, I. (2017): Evaluation of Diagnostic Quality of Chest Radiographs Seen in a Nigerian Teaching Hospital. J. Adv. Med. Med. Res., 23(3): 1

- Okeji, Mc.; Anakwue, A. and Agwuna, K. (2010): Radiation exposure from diagnostic radiography: an assessment of X-ray beam collimation practice in some Nigerian Hospitals. Internet. J. Med. Update, 5(2): 31 . 
- Peter, J.L. (2004): Quality Assurance Workbook for Radiographers \& Radiological Technologists. World Health Organization

- Stollfuss, J.; Schneider, K. and Krüger-Stollfuss, I. (2015): A comparative study of collimation in bedside chest radiography for preterm infants in two teaching hospitals. Eur. J. Radiol. Open, 2: 118. 
(152) 